\title{
TRADUCTION OU NOUVELLE REDACTION? UN EXEMPLE DE DIFFICULTES POSEES PAR LA TRADUCTION TECHNIQUE
}

\author{
Lionel Meney
}

A une époque où la mondialisation de l'économie est en voie de réalisation, ou les frontières politiques sont de plus en plus poreuses, il reste un obstacle de taille à la communication entre les humains, particulièrement entre les producteurs de biens et services et les consommateurs, à savoir les différences linguistiques et culturelles.

On n'a pas encore vraiment étudié, encore moins mesuré, l'influence de la langue et de la culture sur la conception d'un produit comme, par exemple, un ordinateur. Ce type de machine d'utilisation universelle est, comme tout produit, accompagné d'une série de documents--un mode d'emploi--qui représentent le seul lien entre le producteur et le consommateur d'une part, l'utilisateur et la machine d'autre part.

La difficulté de rédiger ce genre de mode d'emploi tient au fait qu'il doit rendre simple, aisément accessible à l'utilisateur qui n'a pas besoin de connaître l'aspect technique, l'utilisation d'une machine et la production d'opérations fondamentalement complexes.

Dans cet article nous allons examiner à partir d'un exemple, le Guide de l'utilisateur' de l'ordinateur personnel IBM, à quel point ces difficultés inhérentes à toute rédaction technique peuvent être augmentées par des problèmes de traduction et de différences culturelles.

Le but de cet opuscule d'environ 170 p. (il n'y a pas de véritable pagination d'un bout a l'autre, mais une pour chacune des sections et des annexes) est de permettre à l'utilisateur de réaliser quelques opérations simples tel que le formatage et la copie de disquette. Nous n'entrerons pas dans le détail des difficultés de compréhension qu'il présente même dans sa version originale anglaise. ${ }^{2}$ Nous insisterons plutôt sur les difficultés supplémentaires dues au fait que nous avons affaire à une traduction, dans une langue, le français, qui n'est pas celle des concepteurs, et dans une culture qui n'a pas forcément les mêmes références ni les mêmes images que la société anglo-saxonne d'Amérique du nord. 
Nous retiendrons trois types de difficultes: celles dues au choix du lexique, celles liées à des problèmes de traduction et celles en relation avec la mise en page de l'ouvrage.

\section{Lexique}

Deux types de difficultés guettent le traducteur technique. Tout d'abord, comme tout rédacteur technique, il doit sans cesse choisir entre l'utilisation du terme technique exact normalement employé par les gens du métier et la nécessité de se mettre au niveau de l'utilisateur qui ne conna ît pas forcément les termes propres, et même qui a de bonnes chances de les ignorer.

Ensuite, dans le cas particulier de notre exemple, l'informatique, nous sommes en présence d'une situation lexicale complexe. En effet, cette industrie plus que d'autres encore, ayant été à ses débuts presque totalement anglo-saxonne, a été à l'origine de la diffusion de très nombreux termes anglais (américains) en français. Au fur et à mesure que l'informatique s'est développée et que les chercheurs et industriels francophones ont assimilé, puis développé eux-mêmes la technique, que les linguistes et terminologues ont réfléchi sur cette langue de spécialité et produit glossaires et lexiques, la langue française s'est enrichie de nombreux néologismes. Avec pour conséquence qu'à côté du jargon des praticiens, fortement anglicisé, existe un jargon des puristes et que l'utilisateur oscille entre les deux...

Les traducteurs du Guide de l'utilisateur ont pris le parti tout à fait louable de franciser au maximum, au risque de produire un texte parfois aseptisé. Cependant il reste encore beaucoup d'anglicismes qui risquent de faire obstacle a la compréhension de l'usager francophone. Ainsi le sigle DOS qui apparaît développé dans la version anglaise: Disk Operating System, ne se trouve que sous cette forme de sigle dans la version française. Donce doublement obscure. D'autant plus que le rapprochement avec l'équivalent français Système d'exploitation n'est pas évident, n'étant plus motivé ni expliqué par le signe linguistique.

Une difficulté d'importance réside dans le fait que les noms des commandes ont été conservés dans leur forme anglaise. Par ex.: Numlock, Home, Prt Sc, etc., ce qui représente plusieurs types de difficultés pour l'usager francophone. A la difficulté de compréhension inhérente à la complexité de la technique s'ajoute la difficulté d'une langue étrangère et de son système particulier d'abréviation. 
Les anglicismes se trouvent également au niveau de certains termes ou de certaines traductions. La plupart du temps ces anglicismes sont entrés dans la langue, du moins dans une certaine langue et il n'est pas question de le reprocher aux traducteurs qui doivent tenir compte de l'usage, qu'il soit critiquable ou non. Voici quelques exemples d'angliscismes lexicaux:-anglicismes par transfert (calque morphologique): éditer sur le modèle de to edit; formater à partir de to format; restaurer sur to restore. Anglicismes par traduction (calque sémantique): bibliothèque sur le modèle de library; répertoire d'après directory; réseau à partir de network.

Dans le premier cas, ce genre d'approximation lexicale est une source réelle d'incompréhension pour l'usager francophone qui a déjà sa propre perception du sens traditionnel des termes tels que éditer ou restaurer. Procédant normalement, comme devant chaque situation nouvelle, du connu vers l'inconnu, il essaie de comprendre le sens de ces termes en contexte à partir de son expérience. Mais dans ce cas, son expérience ne lui est d'aucun secours et le terme ne joue pas son rôle d'intermédiaire entre l'usager et la notion. Parfois, comme dans le cas de formater ${ }^{3}$, l'incompréhension est totale.

Parfois l'anglicisme concerne non pas un terme simple, mais un syntagme. Voici quelques cas de calques syntagmatiques relevés dans le Guide de l'utilisateur: Nom de fichier sur le modèle de Filename; label de volume d'après volume label (calque particulièrement malheureux parce que le mot label en français ne renvoie pas directement à la notion d'uétiquette», de "désignation" mais de "signe», de «symbole» comme on le voit par l'expression «label de qualitè); disquette cible à partir de target diskette, etc.

Un cas particulièrement intéressant est le terme routine, terme d'origine française emprunté depuis longtemps par l'anglais («a regular procedure prescribed as daily life» qui, par extension, a créé un sens nouveau, spécialisé, inconnu du français (et des francophones): «a set of coded instructions for a computer».

Jusqu'à présent nous avons mis l'acent sur l'abondance des anglicismes. Mais ce n'est pas la seule difficulté lexicale du Guide de l'utilisateur (et de bien d'autres ouvrages d'informatique). Un autre problème est lié à l'utilisation de termes techniques. Dans le Guide on n'en compte pas moins de 150. Par ex.: programme d'anorçage, commande interne, commande externe, compatibilité, configuration, par défaut, disque dur, impression en écho, erreur irrémédiable, mémoire tampon, nom réservé, octet, configuration partagée, partition DOS, valide, zone, etc. 
Certes, tous ces termes ne présentent pas la même difficulté de compréhension. Certains tel disque dur, représentant des objets concrets, parlent plus facilement à l'imagination que d'autres tels octet ou mémoire tampon, qui désignent des réalités plus abstraites et complexes. Cependant l'objectif d'un manuel d'utilisation est de faciliter la tâche de l'utilisateur novice. Il conviendrait donc de le faire dès l'étape du choix des termes. Mais à cet égard le traducteur est moins libre que le rédacteur...

Autre cas digne d'être mentionné, le terme configuration qui, en français, signifie «forme générale, aspect d'ensemble». Ce qui n'est pas particulièrement précis...Or, ce mot est en informatique un terme spécialisé. Il désigne la composition d'un système informatique donné (telle unité centrale, tels périphériques, etc.). Mais il peut aussi désigner le type de clavier: clavier anglais (QWERTY) ou français (AZERTY). Autrement dit nous sommes en présence d'un cas de polysémie. La bête noire des terminologues. Imaginez la perplexité de l'utilisateur novice quand il lit à la p. I-17: "Votre configuration peut comporter des unités de disquette et des unités de disque dur» (sens no 1) et à la p. VI-17: «Si la version DOS utilisée ne correspond pas à la configuration du clavier...» (sens no 2)!

Mais le traducteur n'est pas seulement dépendant d'un certain type de vocabulaire: Il est tributaire des qualités et des défauts de rédaction du texte à traduire. Le User's Guide a fait des efforts remarquables d'explication didactique et d'illustration. Cependant on peut regretter qu'il n'y ait pas systematiquement de définition immédiatement à la suite de chaque notion nouvelle. Et ce défaut se retrouve évidemment dans la version française. Parfois la définition est donnée bien après la première occurrence du terme, parfois il n'y a pas de définition du tout.

Maintenant nous allons nous attacher à trois autres exemples de difficultés qui se présentent au traducteur technique.

\section{Traduction des jeux de mots et des images}

Un moyen de faire mieux comprendre une notion complexe est l'utilisation du style didactique. Et un prodécé didactique courant est le jeu de mots à des fins de comparaison. Ainsi dans le User's Guide pour faire comprendre la notion (informatique) de file, les rédacteurs ont imaginé de jouer sur les mots: file, filing cabinet, file folder. Par ex.:

Your Fixed Disk--an Electronic Filing Cabiner ${ }^{4}$ 
When we described files earlier in this book, we compared them to files in a filing cabinet. Each file folder in a filing cabinet contains information on a specific subject. (User's Guide, 6-3).

Et le texte est suivi d'une illustration qui représente le tiroir d'un classeur métallique rempli de dossiers ou de chemises (cf. Annexe 1).

Malheureusement le même jeu de mots est impossible en français et le traducteur est piégé. Voici comment les traducteurs du Guide de l'utilisateur ont rendu le texte cité ci-dessus:

Le disque dur: un classeur électronique

Lorsque au début de ce manuel, nous avons décrit les fichiers, nous les avons comparés aux dossiers d'un classeur. Chaque dossier contient des informations sur un sujet déterminé. (Guide de l'utilisateur, 6-3)

La valeur didactique de la comparaison est évidente en anglais, mais elle se perd dans le passage au français car il y a rupture de parallélisme et de correspondance au niveau des signes linguistiques. En effet, comparez:

$\begin{array}{ll}\text { File } & \text { Fichier } \\ \text { Filing Cabinet } & \text { Classeur } \\ \text { File Folder } & \text { Dossier }\end{array}$

Pour que l'image passe, il aurait fallu que le français posséde des mots du genre: fichier, «classeur à fichier», «dossier à fichier». Mais ce n'est pas le cas...

\section{Rapport Texte/llustration}

Ce probleme de traduction a des incidences sur l'illustration. Car si le traducteur francophone est prisonnier du texte anglais, il est aussi dépendant de l'illustration conçue par un illustrateur anglophone à l'intention d'usagers également anglophones. Nous analyserons deux exemples.

Dans le premier cas (User's Guide et Guide de l'utilisateur, 5-30), il s'agit d'une illustration destinée à faciliter la compréhension de l'opération de comparaison entre deux fichiers pour vérifier si la copie est bien identique à l'original.

Le dessin commun aux deux versions représente un oiseau stylisé (un cacatoès?) agitant à droite et à gauche deux «chemises"(?) ou deux 
«dossiers» (?) identiques (cf. Annexe 2). Dans la version anglaise, l'allusion est limpide: to compare two files est illustré par two file folders. Mais là oú l'anglophone verra two file folders, le francophone voit deux chemises. Il n'y a pas de lien sémantique évident entre deux «fichiers» et deux «chemises»! En français, un fichier est quelque chose de volumineux: «une collection de fiches» ou un «meuble, une boîte, un classeur contenant des fiches».

Dans le second cas, on se heurte a une des conséquences du fait qu'on ait conservé les noms de commande anglais. (cf. User's Guide et Guide de l'utilisateur 6-18). En effet pour faire defiler le nom de tous vos répertoires il vous faut utiliser la commande TREE. L'anglophone fera à coup sûr et sur-le-champ le rapprochement entre le nom de la commande (TREE $=$ arbre) et l'illustration qui représente le mème oiseau montrant un arbre (cf. Annexe 3). Mais il est bien évident que l'illustration devient totalement non-motivée dans la version française...

En conclusion, nous dirons à la suite de $\operatorname{COVACS}^{5}$ que dans bien des cas la corédaction, ou une nouvelle rédaction, est de loin préférable à la traduction quand il s'agit de textes techniques.

Même si la science et la technique sont internationales, elles ne sont pas à l'abri d'influences linguistiques et culturelles. De toute façon, les documents d'accompagnement d'un produit s'adressent toujours à un utilisateur qui, lui, baigne dans une culture donnee et parle une langue déterminée.

Dans le cas de l'informatique, comme dans bien d'autres, il y a suffisamment de spécialistes francophones: ingénieurs, rédacteurs scientifiques et techniques, vulgarisateurs pour qu'on confie la rédaction des manuels d'utilisation à des personnes sensibilisèes aux difficultés rencontrées par les membres de leur culture.

Par ailleurs il faudrait faire un effort supplementaire pour rendre simple ce qui est complexe. Un premier pas dans cette direction serait franchi si l'on portait plus de soin au choix des termes, si l'on accompagnait chaque terme spécialisé d'une définition ou d'un renvoi à un glossaire.

Il conviendrait surtout de faire précéder le manuel d'utilisation d'un préambule qui, avant d'expliquer le comment des opérations ferait une présentation accessible de leur pourquoi.

En effet une machine a toujours pour fonction d'exécuter un certain nombre d'opérations. Dans le cas de l'ordinateur, il conviendrait d'expliquer 
au préalable à l'utilisateur quel genre de tâches sa machine est capable d'exécuter!

Lionel Meney est professeur agrégé et vice-doyen à la Faculté des lettres de l'Université Laval (Québec). 


\section{NOTES}

1. DOS version 3.20. Guide de l'utilisateur par IBM Corp. et Microsoft, Inc. 1986.

2. Disk Operating System Version 3.20. User's Guide by IBM Corp. and Microsoft, Inc. (1986).

3. On a proposé de remplacer formater par initialiser qui ne paraît guère plus heureux. Pourquoi ne pas s'en tenir au terme certes plus général, mais combien plus clair: préparer?

4. C'est moi qui souligne (L.M.).

5. Covacs, Alexandre. «Bilinguisme...». Meta, 24, 1, 1979. 


\section{BIBLIOGRAPHIE}

1. Covacs, Alexandre. «Bilinguisme officiel et double version des lois. Un pis-aller: la traduction, une solution d'avenir: la corédaction", Meta 24, 1, 1979, p. 103-108.

2. Juhel, Denis (et collaborateurs). «Les difficultés traductionnelles du Guide de l'utilisateur. Quelques voies de solution» à paraître dans Le Langage et l'Homme.

3. Meney, Lionel (et collaborateurs). Le Guide de l'utilisateur IBM PC. Difficultés linguistiques et rédactionnelles. Centre international de recherche sur le bilinguisme, Québec, Université Laval, 1987, 18 p.

4. Meney, Lionel (et collaborateurs) «Informatique: Manuels d'utilisateur et difficultés de lecture». Le Langage et l'Homme, Vol. 24 (juin 1989), fasc. 2, p. 126-131.

5. Mepham, Michael (et collaborateurs). «Some Aspects of Vocabulary Acquisition in the DOS 3.2 User's Guide and Reference Manual». Langues et linguistique, 15, 1989, p. 201-232. 
ANNEXE I

\section{Le disque dur : un classeur électronique}

Lorsque au début de ce manuel, nous avons décrit les fichiers, nous les avons comparés aux dossiers d'un classeur. Chaque dossier contient des informations sur un sujet déterminé. Pour certains de ces dossiers, il peut s'agir de notes ayant trait à un projet particulier. $D^{\prime}$ autres peuvent contenir des rapports que votre employeur vous demande de mettre à jour à intervalles réguliers. En outre, les dossiers du classeur peuvent être regroupés par catégorie.

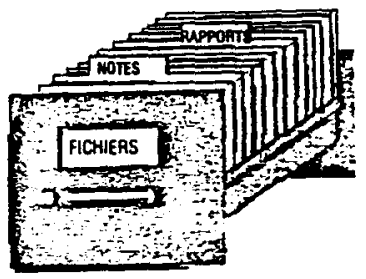


ANNEXE 2

\section{Comment comparer deux fichiers}

Utilisez la commande COMP pour comparer le contenu de deux fichicrs. Cette commande est généralement employée après la commande COPY nour vérifier si la copie est identique à l'original.

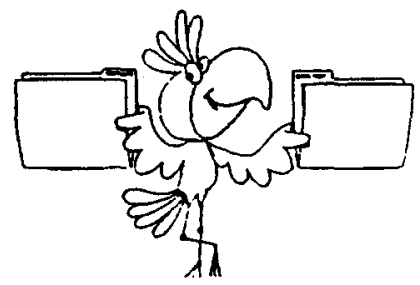


ANNEXE 3

\section{Comment afficher les noms des sous-répertoires}

Vous pouvez demander le nom de tous les sous-répertoires, à l'ajde de la commande TREE. Vous pouvez également obtenir la liste de tous les fichiers, dans chaque sousrépertoire, au moyen de l'option /f.

tree/f

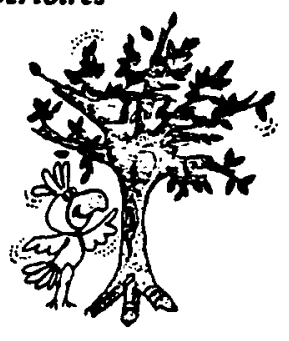

Appuyez sur Entrée.

Pour obtenir la liste des sous-répertoires. sans la liste des fichiers. frappez :

tree

Appuyez sur Entrée. 